\title{
Errores algebraicos en tareas de descomposición factorial por estudiantes universitarios de Nicaragua
}

Algebraic errors in factorial decomposition tasks by university students of Nicaragua

\author{
Sabino Ariel Olivar Molina ${ }^{1}$ \\ William Oswaldo Flores López ${ }^{2}$ \\ Flor Delíz Alvarado Gonzálezz
}

\section{Resumen}

Esta investigación ha analizado los tipos de errores algebraicos, en tareas de descomposición factorial por estudiantes universitarios de Nicaragua. Se trata de un estudio cuantitativo, sustentado en cualitativo por su diseño descriptivo con la participación de 65 estudiantes universitarios de la Universidad Abierta en Línea de Nicaragua. El análisis se basa en la descripción, interpretación y categorización de las respuestas de los educandos. Los principales resultados muestran que los errores algebraicos son de carácter conceptual, procedimental u operacional, lo que se interpreta como un aprendizaje deficiente de las competencias de establecer relación coherente entre los tipos de descomposición factorial y su solución de acuerdo a sus propias características vinculadas a situaciones prácticas de la vida cotidiana. Se concluye, que un error es un conocimiento aprendido insatisfactoriamente y que se puede avanzar en la gestión, diseño e implementación de una comunidad de aprendizaje con incorporación de tecnología para disminuir errores en la resolución de tareas de descomposición factorial.

Palabras clave: Errores algebraicos, descomposición factorial, aprendizaje deficiente, metodologías de enseñanza, comunidad de aprendizaje

\section{Abstract}

This research has analyzed the types of algebraic errors in tasks of factorial decomposition by Nicaraguan university students. It is a quantitative study, based on qualitative by its descriptive design with the participation of 65 university students of the Online Open University of Nicaragua. The analysis is based on the description, interpretation and categorization of the learners' answers. The main results show that algebraic errors are conceptual, procedural or operational, what is interpreted

1 Licenciado en Ciencias de la Educación con Mención en Matemáticas. Profesor de la Universidad de las Regiones Autónomas de la Costa Caribe Nicaragüense-Recinto Universitario Nueva Guinea. Correo: sabino.olivar@uraccan.edu.ni ORCID: https://orcid. org/000o-0003-0505-8757

2 Doctor en Educación. Profesor Investigador de la Universidad de las Regiones Autónomas de la Costa Caribe Nicaragüense. Correo: william.flores@uraccan.edu.ni ORCID: http://orcid.org/oooo-ooo2-1016-1620

3 Máster en Didáctica. Profesora de la Universidad de las Regiones Autónomas de la Costa Caribe Nicaragüense-Recinto Universitario Nueva Guinea. Correo: flor.alvarado@uraccan.edu.ni

Recibido: 01/11/2017 Aprobado: 01/01/2018

Olivar-Molina, S., Flores, W., 0., \& Alvarado-González, F. (2018). Errores algebraicos en tareas de descomposición factorial por estudiantes universitarios de Nicaragua. Revista Electrónica de Conocimientos, Saberes y Prácticas, 1(1), 9-27. D0I: https://doi.org/10.30698/ recsp.vii1.1 
as a deficient learning of the competences of establishing a coherent relationship between the types of factorial decomposition and their solution according to their own characteristics linked to practical situations of daily life. It is concluded that an error is an unsatisfactorily learned knowledge and that one can advance in the management, design and implementation of a learning community with the incorporation of technology to reduce errors in the resolution of factorial decomposition tasks.

Keywords: Algebraic errors, factorial decomposition, poor learning, teaching methodologies, Learning community

\section{Introducción}

Esta investigación trata de analizar los tipos de errores algebraicos en tareas comunes de descomposición factorial por el estudiantado de la Universidad Abierta en Línea de Nicaragua al iniciar sus estudios universitarios. Existe una preocupación por parte del profesorado universitario, por qué los educandos tienen inopia en sus habilidades de resolver tareas de descomposición factorial algebraica, es decir, generalidades pobres acerca de la resolución de tareas de factorización, obstaculizando el desarrollo de su aprendizaje matemático en las asignaturas posteriores, para alcanzar las matemáticas superiores en la universidad. Esta problemática se ha observado en otros países como España (Torres \& Calderón, 200o), México (García, Segovía \& Lupiañez) y Argentina (Pochulu, 2005; Olmedo et al., 2015).

En la literatura ya existen estudios que tratan de clasificar estos errores e identificar sus causas. Olmedo (2009) sugiere, por ejemplo, que los errores se originan por el uso de estrategias de aprendizaje superficiales, de práctica y memorización con escaso nivel metacognitivo. Para Flores y Auzmendi (2016) los errores algebraicos asociados a soluciones de tareas de descomposición factorial se pueden exteriorizar por: las actitudes hacia las matemáticas; las dificultades de interpretación de las letras; las dificultades debidas a la naturaleza del lenguaje algebraico; dificultades que tienen origen en la aritmética; dificultades en el proceso de generalización y errores procedimentales. Investigando en profundidad porque se cometen estos errores puede servir de ayuda para proponer situaciones didácticas que mejoren el aprendizaje.

Consciente del escenario anterior, en esta comunicación el trabajo se centra, particularmente, en identificar los tipos de errores algebraicos en tareas descomposición factorial. Para ello, se aplicó un cuestionario, compuesto por 11 ítems, dicho instrumento evalúa procesos de carácter procedimental u operacional, y procesos de carácter conceptual en tareas de descomposición factorial, así como un protocolo de entrevista cognitiva para investigar a profundidad porque cometen los errores, en la resolución de ejercicios de descomposición factorial. El análisis se efectuó a partir de una metodología descriptiva de los resultados, pero que, concretamente, se basa en el análisis de las respuestas de los estudiantes. Esto ayudó a clasificar los errores que propone la literatura y otros autores que han aportado al tema en estudio. 


\section{Literatura}

\section{Noción de error}

Los errores algebraicos son una posibilidad permanente en la adquisición y consolidación del aprendizaje, forma parte de las producciones del estudiantado durante su fase de aprendizaje; y son datos objetivos que se dan normalmente de forma estable a lo largo de dicho proceso de aprendizaje (Rico, 1995). Socas (1997) es más preciso y considera el error como un indicador de un esquema cognitivo inadecuado en el estudiando. Además, sugiere que el error, da también información sobre dificultades que presentan contenidos específicos de matemáticas. De acuerdo con Popper (1979) los errores son defectos o averías que se producen durante el proceso de construcción del conocimiento o del desarrollo de las estructuras cognitivas, que están en desarrollo continuo (Rico, 1995).

Los errores Funcionan como elementos usuales en el camino hacia el verdadero conocimiento, porque cuando se trata de superarlos se pone en juego el ejercicio de la autocrítica, el sometimiento a prueba del conocimiento adquirido en diversos contextos y las diversas aproximaciones a la realidad construidas por el individuo Es decir, el error observado puede señalar un camino constante en la forma como se presentan los bloques que permitan construir un sólido conocimiento de los procesos algebraicos por parte del estudiantado. Brousseau, Davis y Werner (1986), quienes consideran que el error es a menudo el resultado de grandes concepciones inadecuadas acerca de aspectos fundamentales de las matemáticas, señalan dos vías mediante las que el error puede presentarse: (1) como resultado de la aplicación correcta y crédula de un procedimiento imperfecto sistematizado que se puede identificar con facilidad por el profesor; $y$ (2) cuando el estudiante utiliza procedimientos imperfectos y posee concepciones inadecuadas que no son reconocidas por el profesor.

En definitiva, un error es un procedimiento equívoco que genera un saber y una práctica no satisfactoria, o sea, un error es un conocimiento aprendido insatisfactoriamente que genera resultados negativos en la resolución de una práctica matemática.

\section{Errores comunes en la descomposición factorial de tareas algebraicas}

La investigación de los errores en la factorización son aproximaciones a los errores algebraicos, los estudios de Radatz (1979); Küchemann (1981); Movshovitz et al. (1987); Alonso et al. (1993); Brousseau (1997); Socas (1997); Astolfi (1999); y Molina, Castro y Castro (2007) han permitido una categorización de dichos errores. En la tabla 1, observamos las tipologías de errores, que argumenta los autores anteriormente mencionados. 
Tabla 1: Tipologías de errores en la descomposición factorial.

\begin{tabular}{|c|c|}
\hline Tipología & Categorías \\
\hline Radatz (1979) & $\begin{array}{l}\text { - Errores debidos a la dificultad del lenguaje. } \\
\text { - Errores debido a dificultades para obtener información espacial. } \\
\text { - Errores debidos a un aprendizaje deficiente de hechos, destreza y conceptos previos. } \\
\text { - Errores debidos a la aplicación de reglas o estrategias irrelevantes. }\end{array}$ \\
\hline Küchemann (1981) & $\begin{array}{l}\text { - Errores por el uso y dominio de las letras en contexto algebraico: } \\
\text { - La letra evaluada } \\
\text { - La letra ignorada } \\
\text { - La letra como un objeto } \\
\text { - La letra como una incógnita específica } \\
\text { - La letra como variable } \\
\text { - La letra usada como valor funcional }\end{array}$ \\
\hline Movshovitz et al. (1987) & $\begin{array}{l}\text { - Errores debidos a datos mal utilizados. } \\
\text { - Errores debidos a una interpretación incorrecta de lenguaje. } \\
\text { - Errores debidos a inferencias no válidas lógicamente. } \\
\text { - Errores debidos al uso de teoremas o definiciones deformadas. } \\
\text { - Errores debido a la falta de verificación en la solución. } \\
\text { - Errores técnicos. }\end{array}$ \\
\hline Alonso et al. (1993) & $\begin{array}{l}\text { - Errores en el proceso de generalización } \\
\text { - Errores en distinguir lo particular de lo general. } \\
\text { - Errores en la deducción de métodos y reglas generales } \\
\text { - Errores en la simbolización y representación del lenguaje natural al lenguaje algebraico }\end{array}$ \\
\hline Brousseau (1997) & $\begin{array}{l}\text { - Error a un nivel práctico. } \\
\text { - Error en la tarea. } \\
\text { - Error de técnica. } \\
\text { - Error de tecnología. } \\
\text { - Error de nivel teórico. }\end{array}$ \\
\hline Socas (1997) & $\begin{array}{l}\text { - Errores que tienen su origen en un obstáculo. } \\
\text { - Errores que tienen su origen en la ausencia de sentido. } \\
\text { - Errores que tienen su origen en actitudes afectivas y emocionales hacia las matemáticas. }\end{array}$ \\
\hline Astolfi (1999) & $\begin{array}{l}\text { - Errores debidos a la comprensión de las instrucciones de trabajo dadas. } \\
\text { - Errores que provienen de los hábitos escolares o de una mala interpretación de las expectativas. } \\
\text { - Los errores como resultados de las concepciones de los propios estudiantes. } \\
\text { - Errores ligados a las operaciones intelectuales implicadas. } \\
\text { - Errores debidos a los procesos adoptados. } \\
\text { - Errores debidos a la sobrecarga cognitiva en la actividad. } \\
\text { - Errores que tienen su origen en otra disciplina. } \\
\text { - Errores causados por la complejidad del contenido. }\end{array}$ \\
\hline $\begin{array}{c}\text { Molina, Castro y Castro } \\
(2007)\end{array}$ & $\begin{array}{l}\text { - Errores por el uso del signo igual } \\
\text { - Uso del signo igual de forma unidireccional y no bidireccional. } \\
\text { - Uso del signo igual como un operador } \\
\text { - Uso del signo igual como equivalencia simbólica } \\
\text { - Uso del signo igual como equivalencia de una identidad estática. }\end{array}$ \\
\hline
\end{tabular}

12 REVISTA ELECTRÓNICA de CONOCIMIENTOS, SABERES Y PRÁCtICAS, Volúmen 1, Año 1, No. 1, Enero-Junio, 2018. 
Según Escudero y Domínguez (2014) los errores relacionados con la descomposición factorial de expresiones algebraicas en las cuales pueden utilizar las igualdades notables, también se pone de manifiesto lo descrito anteriormente como es el caso de la expresión: $4 x^{2}-1=(x-1 / 2)(x+1 / 2)$. En estos casos al obtener las raíces del polinomio no se tiene en cuenta el valor del coeficiente principal en su descomposición factorial. La forma de expresar las raíces en la descomposición factorial también dificulta ese tipo de ejercicios. Así en el ejercicio: calcular un polinomio de grado 6 que tenga $x=2$ como raíz quinta, la respuesta $(x+2)^{5}(x+1)$ indica que utiliza de forma errónea el teorema del resto o bien la definición de raíz de un polinomio. Desde el punto de vista de Escudero y Domínguez (2014), "el error puede ser debido sobre todo a la utilización de, el método de Ruffini, en la descomposición factorial de los polinomios, al expresar el factor de la raíz correspondiente" (p. 117). También, en la descomposición factorial de polinomios, al utilizar la regla de Ruffini en polinomios sin término independiente, olvidan sacar factor común a la variable, y consideran el término de grado uno como término independiente. En otras ocasiones realizan operaciones sin lógica matemática alguna como: $x^{2}-1-x^{2}+3 x=(x-1)(x+1)\left(-x^{2}+3 x\right)=(x-1)(x+1) x(-x+3)$ (Escudero \& Domínguez, 2014, p. 123).

Finalmente, hay que resaltar, los errores por ausencia de respuesta, según Godino, Batanero y Font (2003), son las situaciones del estudiantado que deja en blanco las tareas o carece de destrezas y conceptos previos, o bien, contesta de forma espontánea realizando pasos sin coherencia conceptual o de cálculo sin procesar información alguna, sólo por el mero hecho de escribir algo. En la primera situación, son causados por la carencia de aprendizajes relativos a hechos, destrezas y conceptos, que inhiben totalmente el procesamiento de la información e impiden dar una respuesta a la situación. En la segunda, son que no dejan en blanco ningún ejercicio, no son conscientes de su falta de destreza en el tema, creen que lo están haciendo bien, en realidad se crean sus propios argumentos inconsistentes, dando las respuestas más inverosímiles, es más preocupante el alumnado de esta segunda situación que de la primera.

\section{Metodología de investigación}

Esta investigación es de carácter Mixta. Cuantitativa porque este paradigma ayudó al tratamiento de la información de los datos a través de la categorización y la descripción de las propiedades, características y los perfiles de las personas, grupos, comunidades, procesos y objetos o cualquier otro fenómeno que se someta al análisis (Hernández, Fernández \& Baptista, 2010). Cualitativa porque es una actividad sistemática orientada a la comprensión en profundidad de fenómenos educativos y sociales, a la transformación de prácticas y escenarios socioeducativos, a la toma de decisiones y también hacia el descubrimiento y desarrollo de un cuerpo organizado de conocimiento (Bisquerra, 2009). Mientras que su diseño es de naturaleza descriptiva porque trata de describir el estado presente de cualquier situación educativa con 
implicaciones que van más allá de los límites establecidos por los propios elementos estudiados. Bisquerra, 2009).

\section{Participantes}

En esta investigación participaron 65 estudiantes de la Universidad Abierta en Línea de Nicaragua. Cabe mencionar que los participantes son estudiantes de la carrera Licenciatura en Administración de Empresa con Mención en Turismo y Hotelería, son quienes recibieron el curso de matemática básica correspondiente al primer cuatrimestre. La edad media de los participantes fue de 18 años, con un rango comprendido entre los 18 y los 21 años. El 54\% de los participantes fueron mujeres y el $46 \%$ restantes fueron hombres. Para concluir, indicar que el tipo de muestreo que se utilizó ha sido el muestro no probabilístico causal o accidental, que es aquel en el cual el investigador selecciona directa e intencionalmente la muestra, debido fundamentalmente a que tiene fácil acceso a la misma y es representativa de la población (Gil, Rodríguez \& García, 1995; Albert, 2006; Sabariego, 2004).

\section{Instrumentos}

Para recolectar la información se suministró al estudiantado un cuestionario cognitivo compuesto por 11 ítems, dicho instrumento trata de evaluar el conocimiento aprendido sobre la factorización de expresiones algebraicas. Es decir, evalúa la aplicación de conceptos y propiedades para resolver los algoritmos de factorización por parte del estudiantado universitario.

Tabla 2: Cuestionario sobre algoritmos de factorización

\begin{tabular}{|c|c|}
\hline Descomposición factorial & ítems \\
\hline & 1. Descomponer en factores: $a^{2}+2 a$ \\
\hline Factor común & 2. Descomponer en factores: $10 b-5 a+15 a^{3}$ \\
\hline & 3. Descomponer en factores $72 x^{2 a} y^{b}+48 x^{a+1} y^{b+1}+24 x^{a} y^{2 b}$ \\
\hline & 4. Factorizar $1-a^{2}$ \\
\hline Diferencia de cuadrados & 5. Descomponer $49 x^{2} y^{6} z^{10}-a^{12}$ \\
\hline$a^{2}-b^{2}=(a+b)(a-b)$ & 6. Factorizar $\frac{a^{2}}{4}-\frac{b^{4}}{9}$ \\
\hline & 7. Descomponer $a^{2}+2 a+1$ \\
\hline Trinomio cuadrado perfecto & 8. Factorizar: $1-16 a x^{2}+64 a^{2} x^{4}$ \\
\hline & 9. Factorizar: $\frac{1}{4}-\frac{b}{3}+\frac{b^{2}}{9}$ \\
\hline $\begin{array}{l}\text { Trinomio de la forma } \\
\qquad a x^{2}+b x+c\end{array}$ & $\begin{array}{l}\text { 10. Factorizar: } 20 x^{2}+7 x-6 \text {, utilizando la estrategia más } \\
\text { conveniente para usted, recuerde que es un trinomio de la forma } \\
a x^{2}+b x+c\end{array}$ \\
\hline $\begin{array}{c}\text { Polinomio de la forma } \\
x^{3} \pm 3 x^{2} y+3 x y^{2} \pm y^{3}=(x \pm y)^{3}\end{array}$ & $\begin{array}{l}\text { 11. Descomponer el polinomio } a^{9}-18 a^{6} b^{5}+108 a^{3} b^{10}-216 b^{15} \\
\text { sabiendo que es un polinomio de la forma } x^{3} \pm 3 x^{2} y+3 x y^{2} \pm \\
y^{3}=(x \pm y)^{3}\end{array}$ \\
\hline
\end{tabular}

También, se aplicaron entrevistas cognitivas al estudiantado universitario que solucionaron erróneamente el cuestionario sobre algoritmos de factorización con el propósito de profundizar en los aspectos que no quedaron claros en las respuestas brindadas y para complementar la información de algunas cuestiones que fueron

14 | REVISTA ELECTRÓNICA DE CONOCIMIENTOS, SABERES Y PRÁCTICAS, Volúmen 1, Año 1, No. 1, Enero-Junio, 2018. 
consideradas en la misma. Así mismo, con la entrevista se intentó determinar los posibles patrones de error que contenían sus desarrollos.

\section{Procedimientos de administración, enfoque ético y análisis de datos}

La administración del instrumento se realizó por parte de los autores durante el primer semestre 2017. Tenía un carácter anónimo y fue complementado por los sujetos participantes de forma virtual. Con anterioridad a la toma de los datos, se obtuvo tanto el consentimiento previo, libre e informado del estudiantado, profesorado, así como la autorización de quienes administran el programa de Universidad Abierta en Línea de Nicaragua. El análisis se desarrolló a partir de una metodología de análisis de datos basados en criterios en que incluyen aspectos descriptivos de los resultados pero que, fundamentalmente, se basan en el análisis cualitativo de las respuestas, a través de la categorización de las misma, poniendo como énfasis las capacidades observadas de argumentación de las respuestas.

\section{Resultados}

Sabiendo que los errores surgen por una práctica matemática con argumentos incorrectos, además, que una preocupación constante para el profesorado, es por ello, que el estudio de los errores en tareas de descomposición factorial son elementos importantes para la gestión, diseño e implementación de metodologías de aprendizaje para contribuir a disminuir a los errores algebraicos. En este sentido, se presentan a continuación la categorización de errores algebraicos encontrados a partir del proceso de investigación que se ha realizado.

Tabla 3: Errores en la descomposición factorial: factor común ítem 1.

\begin{tabular}{ccc}
\hline Enunciados & Tipos de Errores & $\%$ \\
\hline & $2 a+a^{2}$ & 46,15 \\
& $a^{2}(2+a)$ & 23,07 \\
$a^{2}+2 a$ & $a(a+a)$ & 15,40 \\
& $a(a+2 a)$ & 7,69 \\
& $a(a+2) 2 a^{2}$ & 7,69
\end{tabular}

Sin Argumento

Total 100

Observamos en la tabla 3, que el estudiantado no alcanzó a factorizar correctamente la expresión, porque cometen diferentes tipologías de errores como: $a(a+a)$; $a^{2}+2 a ; a^{2}(2+a) ; a(a+2 a) ; a(a+2) 2 a^{2}$. Un 46,15\% de los estudiantes comete el tipo error de la forma , se puede notar, que este grupo de estudiante tiene un algoritmo de factorización equivalente a la propiedad conmutativa, en concreto, $a+b=b+a$. Según la entrevista cognitiva, los estudiantes manifiestan que aplican esta propiedad conmutativa porque ordenando los términos de esa manera se obtiene la factorización 
equivalente, debido a que no existen coeficientes numéricos y literales semejantes. El error $a^{2}(2+a)$ tiene una frecuencia del $23,07 \%$, el estudiantado no determina que el factor común es, teniendo en cuenta que este permite dividir a todos los términos de la expresión algebraica, se consultó porque la solución, los educandos opinaron que, el factor común, es el coeficiente literal con mayor grado de exponente.

El 15,40\% de los estudiantes se equivocan por desconocimiento de la división de expresiones algebraicas, por ejemplo, $a(a+a)$, se puede observar que el estudiantado encontró que el divisor de la expresión es " $a$ ", entonces divide $a^{2}$, pero no realiza la división de la expresión del segundo término " $a$ ", además, ha ignorado por completo el coeficiente numérico 2, al momento de factorizar. Las afirmaciones de los estudiantes según la entrevista cognitiva, factorizar ésta expresión consiste en trabajar solamente con la letra " $a$ ". En el caso del error $a(a+2 a)$, un $7,69 \%$ lo comete porque, consideran que factorizar expresiones algebraicas, es solamente dividir coeficientes literales, y no expresiones que están compuestas por coeficientes numéricos y literales, por ejemplo, $2 a$. Los estudiantes consideran que, el procedimiento de factor común, solamente se aplican a términos con letras. Finalmente, un 7,69\% de los educandos cometió el error del tipo $a(a+2) 2 a^{2}$, observamos, que los factores $a(a+2)$ es la solución de $a^{2}+2 a$, mientras que el factor $2 a^{2}$ se interpreta como el producto del primer término con el coeficiente numérico del segundo. Los resultados de la entrevista cognitivas revelan que, $2 a^{2}$ el estudiantado cree que también es divisor de $a^{2}+2 a$.

Tabla 4: Errores en la descomposición factorial: factor común ítem 2.

\begin{tabular}{lcc}
\hline Enunciados & Tipos de errores & $\%$ \\
\hline & $5\left(1 b-1 a+3 a^{3}\right)$ & 30,76 \\
$0 b-5 a+15 a^{3}$ & $2 a b-5+3 a^{2}$ & 15,38 \\
& $\left(26+a+3 a^{3}\right)$ & 23,08 \\
& $5\left(1 b-1 a+a^{3}\right)$ & 7,70 \\
& Sin Argumento & 23,08 \\
\hline & Total & 100
\end{tabular}

En la tabla 4, se presenta los resultados del ejercicio del tipo $10 b-5 a+15 a^{3}$, el estudiantado cometió errores del tipo: $2 a b-5+3 a^{2} ;\left(26+a+3 a^{3}\right) ; 5\left(1 b-1 a+3 a^{3}\right) ; 5\left(1 b-1 a+a^{3}\right)$; y sin argumentos. En la solución $5\left(1 b-1 a+3 a^{3}\right)$ el $30,76 \%$ de los educandos en algunos casos dividió correctamente, pero en el caso de dividir 10 por 5, encontraron una solución de 1 . En el resultado $2 a b-5+3 a^{2}, 15,38 \%$ de los estudiantes no realizó bien la división de polinomios. En la respuesta $\left(26+a+3 a^{3}\right)$ el 23,08\% del estudiantado dividió correctamente la parte numérica, sin embargo, cambio el valor de " $b$ " por 6 . Así mismo, ocurrió con la respuesta $5\left(1 b-1 a+a^{3}\right)$, el 7,70\% del estudiantado encontró soluciones de división $10 \div 5=1$ y $15 \div 5=1$. Finalmente, el 23,08 no argumentó su respuesta. 
Tabla 5: Errores en la descomposición factorial factor común ítem 3.

\begin{tabular}{ccc}
\hline Enunciados & Respuestas & $\%$ \\
\hline & $24 x^{a} y^{b}\left(3 x^{a} y+2 x^{+1} y^{+1}+1 x y^{b}\right)$ & 7,70 \\
$72 x^{2 a} y^{b}+48 x^{a+1} y^{b+1}+24 x^{a} y^{2 b}$ & $24 x^{2}\left(3 x^{a}+2 x^{a}-y^{4}+1 y^{4}\right)$ & 15,38 \\
& $24 x^{a} y^{b}\left(3 x^{a}+2 x^{a} y^{4}+1 y^{2 b}\right)$ & 7,70 \\
& $24 x^{a}\left(3 x^{a}+2 x^{a} y^{4}+1 y^{4}\right)$ & 7,70 \\
& $8 x^{2 a} y^{b}+6^{x a+1} y^{b+1}+3 x^{a} y^{2 b}$ & 15,38 \\
& Sin Argumento & 46,14 \\
\hline & & 100 \\
\hline
\end{tabular}

En la tabla 5, observamos que el estudiantado no contesto correctamente el enunciado. En cambio, errores del tipo: $24 x^{a} y^{b}\left(3 x^{a} y+2 x^{+1} y^{+1}+1 x y^{b}\right) ; 24 x^{2}\left(3 x^{a}+2 x^{a}-y^{4}+\right.$ $\left.\left.1 y^{4}\right) ; 24 x^{a} y^{b}\left(3 x^{a}+2 x^{a} y^{4}+1 y^{2 b}\right) ; 24 x^{a}\left(3 x^{a}+2 x^{a} y^{4}+1 y^{4}\right) ; 8 x^{2 a} y^{b}+6^{x a+1}\right) y^{b+1}+3 x^{a} y^{2 b} \quad$, se manifiestan en las respuestas del estudiantado. El error $24 x^{a} y^{b}\left(3 x^{a} y+2 x^{+1} y^{+1}+1 x y^{b}\right)$ lo cometió el 7,70\% del estudiantado, se observa que la división de coeficientes numéricos fue realizada correctamente, sin embargo, en la simplificación de potencias se cometió el error. Así mismo, sucede en la solución el 15,38\% del estudiantado dividió perfectamente los coeficientes numéricos, pero no utilizó correctamente las propiedades de la potencia, además, que se olvidaron que son los factores de dicha expresión. El 7,70\% del estudiantado cometió el error de no aplicar correctamente las propiedades de la potenciación. No obstante, en la solución el 7,70\% del estudiantado ignoró que la letra $y^{b}$ es factor común, lo que conlleva a efectuar una mala aplicación procedimental de la solución. Y, por último, en la solución $8 x^{2 a} y^{b}+6^{x a+1} y^{b+1}+3 x^{a} y^{2 b}$ encontramos una combinación de errores conceptuales y procedimental en la descomposición factorial factor común con 15,38\% del estudiantado. Destacamos que, el 46,14 del estudiantado no argumentó ninguna solución.

Tabla 6: Errores en la descomposición factorial: Diferencia de cuadrados ítem 4

\begin{tabular}{|c|c|c|}
\hline Enunciados & Tipos de errores & $\%$ \\
\hline \multirow{3}{*}{$1-a^{2}$} & $1(a+a) 1(a-a)$ & 76,92 \\
\hline & $1+a=1+b$ & 15,38 \\
\hline & Sin Argumento & 7,70 \\
\hline & & 100 \\
\hline
\end{tabular}

En el enunciado, $49 x^{2} y^{6} z^{10}$ - $a^{12}$ que se detalla en la tabla 7, se encontró que el $61,54 \%$ del estudiantado cometió errores, y el 38,46\% no argumentó una solución. El $15,39 \%$ del estudiantado cree que resolver la expresión $49 x^{2} y^{6} z^{10}-a^{12}$ significa extraer raíces cuadradas como $\left(7 x y^{3} z^{5}-a^{6}\right)$. El 15,39\% del estudiantado piensa que la solución de la expresión $49 x^{2} y^{6} z^{10}-a^{12}$ significa trabajar nada más con las letras y no con los coeficientes numéricos $\left(49 x y^{3} z^{5}-a^{6}\right)\left(49 x y^{3} z^{5}+a^{6}\right.$. Un 7,69\% del estudiantado opina que la solución es escribir las diferencias de cuadrados con la misma expresión original $\left(49 x^{2} y^{6} z^{10}-a^{12}\right)\left(49 x^{2} y^{6} z^{10}+a^{12}\right)$. Otro $7,69 \%$ del estudiantado, ofrecen una respuesta errada del tipo $\left(7 x y^{3} z^{5}\right)\left(7 x y^{3} z^{5}-a^{2}\right)$ entendiéndose como el factor común de 
la expresión, pero equivocándose en su faceta procedimental. Y finalmente, un 7,69\% del estudiantado, se equivocó por mala aplicación de las propiedades de las potencias $\left(7 x y^{3} z^{5}-a^{4}\right)\left(7 x y^{2} z^{2}+a^{3}\right)$.

Tabla 7: Errores en la descomposición factorial: Diferencias de cuadrados ítem 5

\begin{tabular}{ccc}
\hline Enunciados & Tipos de errores & $\%$ \\
\hline & $\left(7 x y^{3} z^{5}-a^{6}\right)$ & 15,39 \\
& $\left(49 x y^{3} z^{5}-a^{6}\right)\left(49 x y^{3} z^{5}+a^{6}\right)$ & 15,39 \\
$49 x^{2} y^{6} z^{10}-a^{12}$ & $\left(49 x^{2} y^{6} z^{10}-a^{12}\right)\left(49 x^{2} y^{6} z^{10}+a^{12}\right)$ & 15,39 \\
& $\left(7 x y^{3} z^{5}\right)\left(7 x y^{3} z^{5}-a^{2}\right)$ & 7,68 \\
& $\left(7 x y^{3} z^{5}-a^{4}\right)\left(7 x y^{2} z^{2}+a^{3}\right)$ & 7,69 \\
& Sin Argumento & 38,46 \\
\hline
\end{tabular}

En la tabla 8, se presenta la descomposición factorial de $a^{2} / 4-b^{4} / 9$, en este enunciado, el estudiantado no argumentó correctamente, es decir, el 100\% se deben argumentos incorrectos y sin argumentos. El 7,69\% del estudiantado cometió errores por uso inadecuado de la potenciación en las letras, por ejemplo: $a / 2-b^{2} / 3=(a / 2+$ $\left.b^{2} / 3\right)\left(a / 2-b^{2} / 3\right)$. Un $23,08 \%$ del estudiantado, no extrajo las raíces cuadradas de los coeficientes numéricos $(a / 4-b / 9)(a / 4+b / 9)$. El 7,69\% no trabajó con la parte procedimental de los signos $(a / 2-b / 3)(a / 2-b / 3)$. El 7,69\% confundió la parte conceptual de diferencias de cuadrados con la de propiedad conmutativa de los números reales, así misma aplicación de propiedades potenciación $\left(a^{2} / 2-b / 3\right)=a / 2+b^{3} / 2$. Mientras que un $15,38 \%$ del estudiantado, planteó una suma de fracciones como $a / 2-b^{2} / 3+a / 2$ - $b^{2} / 3$ olvidándose de la parte conceptual y procedimental y nada más usando igualdades. Un 15,39\% solamente hizo réplicas de las expresiones originales de la manera siguiente $\left(a / 4-b^{2} / 9\right)\left(a^{2} / 4+b^{2} / 9\right)$. Un 15,39\% de las respuestas erróneas se deben a que el estudiantado propone una simplificación de la expresión original y posteriormente una resta, donde se equivoca con la definición de resta de términos semejantes en álgebra $4 a^{2}-9 b^{4}=-5 a b^{2}$. Finalmente, un 7,69\% no argumentó el enunciado.

Tabla 8: Errores en la descomposición factorial: Diferencia de cuadrados ítem 6

\begin{tabular}{ccc}
\hline Enunciados & Tipos de errores & $\%$ \\
\hline & $a \frac{b^{2}}{2}=\left(\frac{a}{2}+\frac{b^{2}}{3}\right)\left(\frac{a}{2}-\frac{b^{2}}{3}\right)$ & 7,69 \\
& $\left(\frac{a}{4}-\frac{b}{9}\right)\left(\frac{a}{4}+\frac{b}{9}\right)$ & 23,08 \\
& $\left(\frac{a}{2}-\frac{b}{3}\right)\left(\frac{a}{2}-\frac{b}{3}\right)$ & 7,69 \\
$\frac{a^{2}}{4}-\frac{b^{4}}{9}$ & $\left(\frac{a^{2}}{2}-\frac{b}{3}\right)=\frac{a}{2}+\frac{b^{3}}{2}$ & 7,69 \\
& $\frac{a}{2}-\frac{b^{2}}{3}+\frac{a}{2}-\frac{b^{2}}{3}$ & 15,38 \\
& $\left.a \frac{b^{2}}{4}-\frac{a^{2}}{9}\right)\left(\frac{b^{2}}{4}+\frac{b^{2}}{9}\right)$ & 15,39 \\
& $4 a^{2}-9 b^{4}=-5 a b^{2}$ & 15,39 \\
& Sin Argumento & 7,69 \\
\hline Total & 100 \\
\hline
\end{tabular}


En el caso del trinomio cuadrado perfecto $a^{2}+2 a+1$, el $46,15 \%$ del estudiantado argumento correctamente el enunciado, mientras que el 30,77\% cometió errores y 23,08 no argumentó. En cuanto, a los errores cometidos por el estudiantado: el 23\% del estudiantado cometió el error de aplicar conceptos y procedimientos para resolver una diferencia cuadrados y no un trinomio cuadrados perfectos obteniendo como resultados $(a+1)(a-1)$. Un 7,69\% del estudiantado aparentemente domina la concepción de factorizar trinomios cuadrados perfectos, sin embargo, no aplicó bien la faceta procedimental, dando como respuesta $(a+2+1)^{2}$.

Tabla 9: Errores en la des composición factorial: Trinomio cuadrado perfecto ítem 7

\begin{tabular}{|c|c|c|}
\hline Enunciados & Respuestas & $\%$ \\
\hline \multirow[b]{2}{*}{$a^{2}+2 a+1$} & $(a+1)(a-1)$ & 46,15 \\
\hline & $(a+2+1)^{2}$ & 30,77 \\
\hline & & 100 \\
\hline
\end{tabular}

Para la descomposición factorial relacionada con la expresión 1-16ax ${ }^{2}-64 a^{2} x^{4}$ se encontró que el 53,84\% cometió errores y el 46,16\% no argumentó. Véase la tabla 9. Los errores relacionados con la expresión 1-16ax $x^{2}-64 a^{2} x^{4}$, se deben a que el estudiantado aplicó incorrectamente el procedimiento factorización de trinomio cuadrado perfecto, marcado por una regla de diferencia de cuadrados y factor común para los casos $1-\left(8 a x-32 a x^{2}\right)$ con $7,69 \%$ y el caso $1-\left(8 a x-32 a x^{2}\right)$ con $15,39 \%$. Mientras que un $7,69 \%$ del estudiando aplicó una transformación de los signos en la expresión original obteniendo como resultado $64 a^{2} x^{4}-16 a x^{2}-1$. Otro 7,69\% del estudiantado dividió algunos factores por 4 obteniendo como resultado una nueva expresión 1-4ax-8ax ${ }^{2}$, así mismo aplicaron factor común a los coeficientes numéricos 16 y 64 . En cambio, un 7,69\% del estudiantado desarrolló la expresión, obteniendo como resultado $\left(1+4 a x^{2}\right)^{2}$ cometiendo el error de extraer raíz cúbica al coeficiente numérico 64. Finalmente, decir, que el 46,16\% del estudiantado no argumentó.

Tabla 10: Errores en la descomposición factorial: Trinomio cuadrado perfecto ítem 8

\begin{tabular}{lcc}
\hline Enunciados & Tipos de errores & $\%$ \\
\hline & $1-\left(8 a x-32 x^{2}\right)$ & 15,38 \\
& $64 a^{2} x^{4}+16 a x^{2}-1$ & 7,69 \\
$1-16 a x^{2}+64 a^{2} x^{4}$ & $4\left(1-4 a x-8 a x^{2}\right)$ & 7,69 \\
& $1-\left(8 a x-32 a x^{2}\right)$ & 15,39 \\
& $\left(1+4 a x^{2}\right)^{2}$ & 7,69 \\
& Sin Argumento & 46,16 \\
\hline & & 100 \\
\hline
\end{tabular}

En relación con el trinomio cuadrado perfecto $1 / 4-b / 3+b^{2} / 9$ se encontró que el $61,53 \%$ cometieron errores y $38,47 \%$ no argumentó ninguna respuesta. Los errores comunes, se destaca que el $23,08 \%$ del estudiantado suma los términos que no son 
semejantes para reducir el enunciado a una sola expresión, es decir, $\left(1 b^{3}\right) / 10$. Un 7,69\% aplica los procedimientos de extracción de raíces al enunciado, pero se olvida del resto de los procedimientos $1 / 2-b / 3$. Otro $7,69 \%$ del estudiantado configura un recíproco de la forma $1 / 2-b / 1+b / 3^{2}=(1 / 2+b / 1-b / 3)^{2}$ sin tener alusión alguna a los procedimientos de descomposición factorial de un trinomio. En cambio, un 7,69\% del estudiantado comete el error de descomponer factorialmente la primera expresión, sin embargo, extrae las raíces cuadradas de la segunda, pero no finalizan el procedimiento de trinomio cuadrado perfecto, obteniendo como resultado $1 b / 1-b / 3$. Finalmente, un $7,69 \%$ configura unas igualdades con la suma de fracciones en los denominadores $-1 b$ / $(43+9)=\left(1 b^{2}\right) /(21+3)$. Véase la tabla 10.

Tabla 11: Errores en la descomposición factorial: Trinomio cuadrado perfecto ítem 9

\begin{tabular}{lcc}
\hline Enunciados & Tipos de errores & $\%$ \\
\hline & $\frac{1 b^{3}}{10}$ & 30,77 \\
& $\frac{1}{\frac{1}{2}-\frac{b}{3}}$ & 7,69 \\
$\frac{1}{4}-\frac{b}{3}+\frac{b^{2}}{9}$ & $\frac{1}{2}-\frac{\mathrm{b}^{2}}{1}+\frac{\frac{2}{3}^{2}}{2}=\left(\frac{1}{2}+\frac{\mathrm{b}}{1}-\frac{\mathrm{b}}{3}\right)^{2}$ & \\
& $\frac{1 b}{1}-\frac{b}{3}$ & 7,69 \\
& $-\frac{1 b^{2}}{43+9}=\frac{1 b^{2}}{21+3}$ & 7,69 \\
& Sin Argumento & 7,69 \\
\hline Total & 38,47 \\
\hline
\end{tabular}

En la descomposición factorial del trinomio de la forma $20 x^{2}+7 x-6$ se encontró que un $92,31 \%$ de errores y $7,69 \%$ de argumentos sin responder. El error frecuente en esta descomposición factorial es el siguiente $2 x+1 x-3=2+1 x-3$, el 92,31\% del estudiantado emplea un procedimiento de división de la expresión original, aunque un poco errado, es decir, dividieron por parte a la expresión algebraica original: por ejemplo: $20 \div 10=2 ; 7 \div 7=1 ; 6 \div 2=3$ posteriormente, anexaron el signo igual, obteniendo dos igualdades distintas.

Tabla 12: Errores en la descomposición factorial trinomio de la forma ítem 10

\begin{tabular}{ccc}
\hline Enunciados & Tipos de errores & $\%$ \\
\hline \multirow{2}{*}{$20 x^{2}+7 x-6$} & $2 x+1 x-3=2+1 x-3$ & 92,31 \\
& Sin Argumento & 7,69 \\
\hline & & 100 \\
\hline
\end{tabular}

En la descomposición factorial del polinomio de la forma $x^{3} \pm 3 x^{2} y+3 x y^{2} \pm y^{3}=(x \pm y)^{3}$, se presentó al estudiantado un ejercicio sencillo de la forma $a^{9}-18 a^{6} b^{5}+108 a^{3} b^{10}$ $216 b^{15}$, el 84,60\% cometió errores y 15,40 no argumentó nada. En la tabla 12, observamos que el $84,60 \%$ del estudiantado comete el error de proponer como solución dos igualdades en la solución de la expresión original, indicando el poco dominio de este procedimiento factorización. 
Tabla 13: Errores en la descomposición factorial: Polinomio de la forma ítem 11

\begin{tabular}{cc}
\hline Enunciados & Tipos de errores \\
\hline & $a^{6}-9 a^{3} b^{4}+12 a b^{6}-54 b^{9}$ \\
$-18 a^{6} b^{5}+108 a^{3} b^{10}-216 b^{15}$ & $=a-9 a^{3} b^{2}+12 a b^{3}-54 b^{6}$ \\
& Sin Argumento
\end{tabular}

Total

\section{Discusión}

Los errores en la descomposición factorial factor común se caracterizan por desconocimientos de conceptos, propiedades y procedimientos de división de expresiones algebraicas e ignoran los coeficientes numéricos en la división, esto se debe porque el estudiantado aplica reglas o estrategias irrelevantes (Radatz, 1979); aplicación equivocadamente de la propiedad conmutativa, este error es producto de la experiencia previa de los estudiantes y del procesamiento interno de estas experiencias (Brousseau, 1996). Los estudiantes relacionan la descomposición factorial factor común con la letra de mayor grado de exponente, este error se manifiesta por interpretar incorrectamente los datos subyacentes a la expresión (Movshovitz et al., 1987). También, le suelen dar valores numéricos a las letras y en ocasiones la ignoran las letras cuando son factores comunes, esto se relaciona con los errores en el uso y dominio de las letras en contexto algebraicos (Küchemann, 1981). Finalmente, existen errores asociados con la aplicación de propiedades de la potenciación que tienen su origen en la aritmética (Socas, 1997), es decir, en la aplicación de procedimientos operacionales y conceptuales relacionados con las propiedades de los números reales.

En la descomposición factorial diferencia de cuadrados se caracterizan, en primer lugar, por obstáculos cognitivos (Brousseau, 1997) como falta de dominio al extraer raíces cuadradas; errores en la aplicación de las propiedades de la potencia; y aplican erradamente la propiedad conmutativa para descomponer las expresiones. También, existen errores de origen aritméticos (Socas, 1997) en el contexto procedimental u operacional en el desarrollo de la adición y simplificación de expresiones fraccionarios; y el desconocimiento de la resta de términos semejantes en expresiones algebraicas. Así mismo, se encontraron errores por uso y dominio de letras (Küchemann, 1981), por ejemplo, el estudiantado ignora los coeficientes numéricos de la expresión y usan incorrectamente los símbolos (Davis, 1975). Por otra parte, surgen errores como la repetición de la misma expresión matemática, aplicación de la descomposición de factor común en una situación de diferencia de cuadrados y usan equivalencias erradas a los factores en la descomposición factorial algebraica.

En la descomposición factorial trinomio cuadrado perfecto se registran errores operacionales: simplificación de expresiones algebraicos no semejantes; descomponen la expresión por parte, sin finalizar el procedimiento factorial, estos errores se manifiestan por no entender la generalización de las relaciones y los procesos 
matemáticos (Socas, 1997). También se hallaron errores procedimentales, es decir, aplican procedimientos de descomposición factorial factor común y diferencias de cuadradas; así mismo, transforman las expresiones algebraicas con signos cambiados erróneamente, y factorizan los coeficientes numéricos ignorando el papel de las letras en álgebra. Estos errores procedimentales coinciden con los encontrados en los estudios de Socas (1997), caracterizándose por usar inapropiadamente fórmulas, reglas y procedimientos algebraicos, así mismo, estos errores concuerdan con los estudios de Küchemann (1981) desde el punto de vista, que las letras son ignoradas porque el estudiantado trabaja la parte de los coeficientes numéricos olvidando aplicar procedimientos algebraicos a las letras. Además, existen errores conceptuales como extraer raíces cúbicas, en vez, de extraer la raíz cuadrada; y configurar igualdades con la suma de los denominadores, relacionando con los errores en la aplicación del concepto y la técnica correcta de Brousseau (1997).

Los errores en la descomposición factorial trinomio de la forma se manifiestan por uso incorrectamente de las estrategias de descomposición factorial, por ejemplo, el estudiantado divide la expresión algebraica en diferentes divisores, olvidándose de los siguientes procedimientos: que extraer raíces cuadradas en el primer término, dividir por el factor ; definir el signo de la expresión algebraica; definición de dos números que den el valor absoluto de ; y simplificar la expresión. Este error se relaciona con los errores por aplicaciones de reglas o estrategias irrelevantes encontrado en los estudios de Radatz (1979). En correspondencia con las respuestas sin argumento, se manifiestan por debilidades en sus habilidades metacognitivas, así como expresa Godino et al. (2003) son carencias de destreza previas, o como afirma Brousseau (1997) es un obstáculo cognitivo, es decir, un conocimiento matemático aprendido, pero al momento de su aplicación ha sido olvidado por el estudiantado.

Finalmente, los errores en la descomposición factorial del polinomio de la forma $x^{3} \pm 3 x^{2} y+3 x y^{2} \pm y^{3}=(x \pm y)^{3}$, el estudiantado no aplica el concepto, las estrategias y procedimientos de la descomposición factorial, es decir: identificar si tiene cuatro términos; que el primer y el último término sean cubos perfectos o que existen dos términos con cubos perfectos; que el segundo término sea más o menos el triple del cuadrado de la raíz cúbica del primer término multiplicado por la raíz cúbica del último término; y que el tercer término sea más el triple de la raíz cúbica del primer término por el cuadrado de la raíz cúbica del último. Si todos los términos de la expresión son positivos, la expresión dada es el cubo de la suma de las raíces cúbicas de su primer y último términos, y si los términos son alternativamente positivos y negativos la expresión dada es el cubo de la diferencia de dichas raíces (Baldor, 2008, p. 167).

Por consiguiente, surge errores conceptuales, procedimentales u operacionales, por ejemplo, reescribir la solución en igualdades idénticas, este error conceptual, procedimental u operacional se relaciona con las ideas de Socas (1997) en el sentido, de errores por el uso de recíprocos, además con los estudios de Vega-Castro, Molina, Castro 
(2012) desde la perspectiva estructural que implica solucionar tareas de simplificación de fracciones algebraicos que involucran igualdades notables. Sobre las respuestas $\sin$ argumentos, son errores debidos a un aprendizaje deficiente de hechos, destreza y conceptos previos, este tipo de error surge por las deficiencias de conocimiento sobre contenidos y procedimientos específicos para la realización de una tarea matemática. Estas deficiencias, incluyen la ignorancia de los algoritmos incorrectos, conocimiento inadecuado de hechos básicos, procedimientos incorrectos en la aplicación de técnicas y dominio insuficiente de símbolos y conceptos necesarios (Rico, 1995).

\section{Conclusiones}

El presente trabajo se ha centrado en analizar los tipos de errores algebraicos en tareas descomposición factorial por el estudiantado de la Universidad Abierta en Línea de Nicaragua al iniciar sus estudios universitarios. En particular, se ha estudiado las razones que conllevan al estudiantado a cometer dichos errores, obteniendo una tipología que engloba los errores algebraicos identificados por otros autores (Davis, 1975; Movshovitz et al., 1987; Rico, 1995; Brousseau, 1997; Socas, 1997; Astolfi, 1999; Godino, Batanero y Font, 2003; Molina et al., 2007; Vega-Castro et al., 2012; Escudero y Domínguez, 2014) y que incluyen otros no identificados en la literatura. La revisión de la literatura identificó los errores más comunes que cometen los alumnos al resolver expresiones algebraicas y derivó una posible clasificación de los mismos. La mayoría de los errores se replicaron en el estudio, aunque se identificó un número importante de causas de dichos errores que no habían sido registradas en los estudios previos.

En este sentido, los tipos de errores que comete el estudiantado universitario en la resolución de tareas de descomposición factorial están asociadas con el concepto, propiedades y reglas definidas para la solución de la descomposición factorial en álgebra. Lo que se interpreta como un aprendizaje deficiente de las competencias de establecer relación coherente entre los tipos de descomposición factorial y su solución de acuerdo a sus propias características vinculadas a situaciones prácticas de la vida cotidiana. Estas dificultades de aprendizaje se pueden entender como aquel conocimiento que ha sido en general satisfactorio durante un tiempo para la resolución de una práctica matemática, y que por esta razón se fija en la mente del estudiantado, pero que posteriormente este conocimiento resulta inadecuado y difícil de adaptarse cuando el estudiante se enfrenta a nuevos problemas (Brousseau, 1997).

Se concluye, que un error es un conocimiento aprendido insatisfactoriamente y que se puede avanzar en la gestión, diseño e implementación de una comunidad de aprendizaje con incorporación de tecnología para disminuir errores en la resolución de tareas de descomposición factorial por estudiantes universitarios. 
A continuación, se presentan los tipos de errrores algebraicos en tareas de descomposición factorial. Veáse tabla 14.

Tabla 14: Errores en la descomposición factorial algebraica

\begin{tabular}{|c|c|}
\hline $\begin{array}{l}\text { Descomposición } \\
\text { factorial }\end{array}$ & Tipo de Errores \\
\hline Factor común & $\begin{array}{l}\text { - } \quad \text { Desconocimiento de conceptos, propiedades y procedimientos de división de } \\
\text { - } \quad \text { Ignoresiones algebraicas. } \\
\text { - }\end{array}$ \\
\hline Diferencia de cuadrados & $\begin{array}{l}\text { - } \quad \text { Falta de dominio de extraer raices cuadradas. } \\
\text { - } \quad \text { Errores en la aplicación de las propiedades de la potencia. } \\
\text { - } \quad \text { expres en la aplicación de la propiedad comuntativa para descomponer } \\
\text { - } \quad \text { Errores en la simplificación de expresiones fraccionarias. } \\
\text { - } \quad \text { Desconocimiento del procedimiento de resta de expresiones algebraicas con } \\
\text { - términos semejantes. } \\
\text { - Errores por ignorar las letras. } \\
\text { - Uso incorrectamente de los símbolos. } \\
\text { - } \quad \text { Aplicación de la descomposición factorial factor común en una situación de } \\
\text { - } \quad \text { diferencia de cuadrados. }\end{array}$ \\
\hline Trinomio cuadrado perfecto & $\begin{array}{l}\text { - } \quad \text { Simplificación de expresiones algebraicas no semejantes. } \\
\text { - } \quad \text { Error por descomposición de la expresiones algebraica por parte. } \\
\text { - } \quad \text { Errores por aplicación de procedimientos de factor comun y diferencias } \\
\text { - } \quad \text { Errodrados. } \\
\text { - } \quad \text { Errores por transformar las expresiones descomponer factorialmente los coeficientes numéricos } \\
\text { ignorando las letras. } \\
\text { - } \quad \text { Errores por extraer raices cúbicas, en situaciones de extraer raices cuadradas. } \\
\text { - } \quad \text { Errores por configurar igualdades con la suma de los denominadores. }\end{array}$ \\
\hline $\begin{array}{l}\text { Trinomio de la forma: } \\
\qquad a x^{x}+b x+c \text { : }\end{array}$ & $\begin{array}{l}\text { - Uso incorrecto de las estrategias y procedimientos de la descomposición } \\
\text { factorial. } \\
\text { - Errores por dividir la expresión algebraica en diferentes divisores. } \\
\text { - } \quad \text { Errores por debilidades en sus habilidades metacognitivas. }\end{array}$ \\
\hline $\begin{array}{l}\text { Polinomio de la forma: } \\
x^{3} \pm 3 x^{2} y \pm 3 x y^{2} \pm y^{3}\end{array}$ & $\begin{array}{l}\text { - Errores por reescribir la solución en igualdades idénticas } \\
\text { - Errores por un obstáculo cognitivo. } \\
\text { - Errores por desconocimiento del procedimiento de descomposición factorial, } \\
\text { es decir, dejan sin argumento las respuesta. }\end{array}$ \\
\hline
\end{tabular}




\section{Lista de referencias}

Albert, M. (2006). La investigación educativa. Claves Teóricas. Madrid: McGraw-Hill.

Alonso, F., Barbero, C., Fuentes, I., Azcárate, A., Dozagarat, J., Gutierrez, S., Riviére, V., Veiga, C. (1993). Ideas para enseñar álgebra. Madrid: Editorial Síntesis.

Astolfi, J. (1999). El error un medio para enseñar. España: DIADA Editora SL.

Baena-González M., M., y Chica Merino, E. (2014). Desarrollo de la competencia matemática en alumnos de Magisterio a través del aprendizaje práctico. Aula de Encuentro, 16(1), 71-88.

Baldor, A. (2008). Álgebra. México: Grupo Editorial Patria.

Bisquerra, R. (2009). Metodología de la investigación educativa. Madrid: La Muralla.

Brousseau, G. (1997). Theory of Didactical Situations in Mathematics. Dordrecht, Países Bajos: Kluwer

Brousseau, G., Davis, R., \& Werner, T. (1986). Observing Student at work. In B. Christiansen, A., G. Howson, \& M. Otte. (Eds.). Perspetives on Mathematics Education. Dordrecht: Reidel Publishing Company.

Davis, R. (1975). Cognitive processes involved in solving simple algebraic equations. Journal of Children's Mathematical Behavior, 1(3), 7-35.

Escudero, A., M., \& Domínguez, J. (2014). De los errores identificados en la investigación a los errores encontrados en un aula de primero bachillerato. Números. Revista de Didáctica de las Matemáticas, 86, 111-130.

Flores, W., O., \& Auzmendi, E. (2016). Los problemas de comprensión del álgebra en estudiantes universitarios. Ciencia en Interculturalidad, 19(2), 54-64. DOI: http:// dx.doi.org/10.5377/rci.v19i2.3119

Flores, W., O., \& Rojas, N. (2013). Integración de las tecnologías de la información y comunicación en la enseñanza universitaria. Horizontes pedagógicos, 15(1), 46-57.

García, J., Segovia, I., \& Lupiáñez, J. (2014). El uso de las letras como fuente de errores de estudiantes universitarios en la resolución de tareas algebraicas. Bolema, 28(50), 1545-1566. http://dx.doi.org/10.159o/1980-4415v28n5oa26 


\section{INVESTIGACIONES EDUCATIVAS}

Gil, J., Rodríguez, G., \& García, E. (1995). Estadística básica aplicada a las ciencias de la educación. Sevilla: Kronos.

Godino, J., Batanero, C. \& Font, V. (2003). Fundamentos de la enseñanza y aprendizaje de la matemática para maestros. Granada: Universidad de Granada.

Hernández, R., Fernández, C., \& Baptista, M. (2010). Metodología de la investigación. México: McGra-Hill.

Küchemann, D. (1981). Children's understanding of numerical variables. Mathematics in school, 7(4), 23-26. http://www.jstor.org/stable/30213397

Molina, M., Castro, E. \& Castro, E. (2007). Historia del signo igual. Granada: Universidad de Granada.

Movshovitz-Hadar, N., Zaslavsky, O., \& Inbar, S. (1987). An empirical classification model for errors in high school Mathematics. Journal for Research in Mathematics Education, 3-14.

Olmedo, N. (2009). Categorización de las ecuaciones a partir del estudio de estrategias de aprendizaje aplicados por alumnos de primer año de la FACEyN. Debates, reflexiones e interrogantes en la educación en ciencias. Argentina: Universidad Nacional de Catamarca.

Olmedo, N., Galíndez, M., Peralta, J., \& Di Barbaro, M. (2015). Errores y concepciones de los alumnos en álgebra. En T. Gutierrez (Ed.). XIV Conferencia interamericana de educación matemática (1-13). México: CIAEM.

Popper, K. (1979). El desarrollo del conocimiento científico. México: Siglo XXI.

Radatz, H. (1979). Error analysis in Mathematics education. Journal for Research in Mathematics Education, 9, 163-172. http://www.jstor.org/ stable/748804?seq=1\#page_scan_tab_contents

Rico, L. (1995): Educación Matemática. En J. Kilpatric, P. Gómez, \& L. Rico (Ed.). Educación Matemática. Errores y dificultades de los estudiantes. Resolución de problemas, evaluación, historia (89-108). Bogotá: Iberoamericana.

Sabariego, M. (2004). El proceso de investigación. En R. Bisquerra (Ed.). Metodología de la investigación educativa (127-163). Madrid: La Muralla. 
Socas, M. (1997). Dificultades y errores en el aprendizaje de las Matemáticas en la Escuela Secundaria. En L. Rico (Ed.). La educación Matemática en la enseñanza secundaria (125-154). Barcelona: ICE-Horsori.

Vega-Castro, D., Molina, M., \& Castro, E. (2012). Sentido estructural de estudiantes de bachillerato en tareas de simplificación de fracciones algebraicas que involucran igualdades notables. Revista Latinoamericana de investigación en matemática educativa, 15(2), 233-258. 